\title{
Summary of the National Advisory Committee on Immunization (NACl) Statement Update on the Recommended Use of Hepatitis A Vaccine
}

\author{
Henry $\mathrm{B}^{1}$, Baclic $\mathrm{O}^{2}$ on behalf of the National Advisory Committee on Immunization (NACl)*
}

\begin{abstract}
Background: The severity of hepatitis A (HA) increases with age. Children less than six years of age are commonly asymptomatic or present with mild disease without jaundice and represent an important source of infection, particularly for household members and other close contacts. In older children and adults, HA is typically symptomatic. Older persons and individuals with chronic liver disease and immunocompromising conditions have an increased risk of progressing to fulminant hepatic failure resulting in death. Immunization with HA vaccine is recommended for pre-exposure immunization of persons at increased risk of infection or severe $\mathrm{HA}$, as well as within 14 days of HA exposure for: susceptible household and close contacts of proven or suspected cases of HA; co-workers and clients of infected food handlers; and staff and attendees of group child care centres and kindergartens where HA has occurred. Canada's National Advisory Committee on Immunization (NACl) has previously recommended HA vaccination for persons one year of age and over.
\end{abstract}

Objectives: To make recommendations for the use of HA vaccine in infants less than one year of age and to clarify recommendations for the post-exposure use of human immune globulin (lg).

Methods: The NACI Hepatitis Working Group (HWG) performed literature reviews and reviewed vaccine manufacturer provided data on the topic of HA post-exposure prophylaxis. All evidence was rated and reported in evidence tables. A knowledge synthesis was performed and $\mathrm{NACl}$ approved specific evidence-based recommendations, elucidating the rationale and relevant considerations.

Results: No studies on the efficacy or effectiveness of HA-containing vaccines in children six to less than 12 months of age were identified through the literature search. Receipt of two doses of HA-containing vaccines was found to be safe and immunogenic in infants six to 12 months of age. Limited data were available regarding HA-containing vaccine immunogenicity in adults over the age of 40 years.

Conclusion: There are now new NACl recommendations on $\mathrm{HA}$ vaccine and post-exposure use of lg.

Suggested citation: Henry B, Baclic O on behalf of the National Advisory Committee on Immunization (NACl). Summary of the National Advisory Committee on Immunization (NACl) Statement Update on the Recommended Use of Hepatitis A Vaccine. Can Comm Dis Rep 2016;42:193-4. https://doi.org/10.14745/ccdr.v42i09a07

\begin{abstract}
Affiliations
${ }^{1}$ National Advisory Committee on Immunization (NACl), Hepatitis Working Group Chair, Victoria, BC

${ }^{2}$ Centre for Immunization and Respiratory Infectious Diseases, Public Health Agency of Canada, Ottawa, ON
\end{abstract}

*Correspondence: naci-ccni@ phac-aspc.gc.ca

\section{Introduction}

The severity of hepatitis $A(H A)$ increases with age. Children less than six years of age are commonly asymptomatic or present with mild disease. In older children and adults, HA is typically symptomatic. Older persons and individuals with chronic liver disease and immunocompromising conditions have an increased risk of progressing to fulminant hepatic failure resulting in death. Immunization with HA vaccine is recommended for pre-exposure immunization of persons at increased risk of infection or severe $\mathrm{HA}$, as well as within 14 days of HA exposure for: susceptible household and close contacts of proven or suspected cases of
HA; co-workers and clients of infected food handlers; and staff and attendees of group child care centres and kindergartens where HA has occurred.

Canada's National Advisory Committee on Immunization (NACl) has previously recommended pre-exposure $\mathrm{HA}$ vaccination for persons one year of age and over. The NACl Hepatitis Working Group (HWG) has recently completed its work on the development of recommendations for the use of HA vaccine in infants less than one year of age and to clarify recommendations for the post-exposure use of human immune globulin (lg). To do this it performed literature reviews and reviewed vaccine 
manufacturer provided data on the topic of HA post-exposure prophylaxis. All evidence was rated and reported in evidence tables. A knowledge synthesis was performed and $\mathrm{NACl}$ approved specific evidence-based recommendations, elucidating the rationale and relevant considerations.

The review of the literature on the use of HA vaccine and current $\mathrm{HA}$ vaccine recommendations are published in the full $\mathrm{NACl}$ statement update (1) and the HA chapter of the Canadian Immunization Guide (2). In summary, no studies on the efficacy or effectiveness of HA-containing vaccines in children six to less than 12 months of age were identified through the literature search. Receipt of two doses of HA-containing vaccines was found to be safe and immunogenic in infants six to 12 months of age. Limited data were available regarding $\mathrm{HA}$-containing vaccine immunogenicity in adults over the age of 40 years. The key recommendations are summarized below.

\section{NACI Recommendations on hepatitis $A$ vaccine and post-exposure use of immunoglobulin}

Recommendation 1: HA vaccine may be provided, beginning at six months of age, to infants who are at increased risk of infection or severe HA. (NACl Recommendation Grade B)

Infants at increased risk of severe HA infection may include those with an underlying liver disease of idiopathic, metabolic, infectious or cholestatic etiology. Canadian-born infants travelling to HA-endemic countries, including children of new Canadians returning to their country of origin to visit friends and relatives may be at increased risk of HA infection.

Recommendation 2: HA vaccine may be provided to infants beginning at six months of age, who are living in a household with an individual who is at increased risk of infection or severe HA. (NACl Recommendation Grade B)

Recommendation 3: For post-exposure prophylaxis, unless contraindicated or unavailable, HA vaccine is recommended in preference to lg for healthy individuals six months of age and older. (NACl Recommendation Grade B)

Because the HA antibody content of Ig is assumed to decrease over time as a result of lower population-level antibody levels (due to lower rates of natural infection), and because of an excellent safety profile of inactivated HA-containing vaccine, immunization is preferred over the administration of a blood-derived product.

Recommendation 4: Immunization with HA vaccine may be considered for all individuals receiving repeated replacement of plasma-derived clotting factors. (NACl Recommendation Grade I)

The solvent-detergent (S/D) method used to prepare plasma-derived clotting factor concentrates does not reliably inactivate the HA virus. However, historically there has been no evidence of HA transmission from plasma-derived clotting factor in Canada and the risk of transfusion-related HA is extremely low because all pooled plasma is tested for HA. Due to a theoretical possibility of infection, immunization of individuals receiving large quantities of plasma-derived clotting factors may be considered. In Canada, product monographs of all
S/D plasma-derived products used in the treatment of conditions requiring clotting factor substitution include recommendations for HA immunization.

Recommendation 5: For post-exposure prophylaxis within 14 days of exposure of susceptible adults 60 years of age and older who are household or close contacts of a case, lg may be provided in addition to HA vaccine. (NACl Recommendation Grade I)

Individuals without a history of disease or previous immunization are susceptible to HA infection. Evidence is suggestive of reduced immunogenic response to $H A$ vaccine, as well as of higher HA infection-related hospitalization and case fatality rates with increasing age. However, due to significant uncertainty about the incremental value of passive immunization on disease outcomes (including Ig HA antibody content), high HA antibody prevalence in older age groups and a small number of cases of HA infection-related complications in individuals over 60 years of age, the decision to include Ig for post-exposure HA prophylaxis should be made on a case-by-case basis. Given the lack of data to support benefit of Ig after 14 days, there is no recommendation for its use after this time period. Post-exposure prophylaxis with vaccine alone is recommended for outbreak response.

Recommendation 6: For post-exposure prophylaxis of susceptible individuals with chronic liver disease, Ig should be provided within 14 days of exposure in addition to HA vaccine. (NACl Recommendation Grade B)

Because of the risk of severe disease and a suboptimal immune response to $\mathrm{HA}$ vaccine among individuals who are immunocompromised and with chronic liver disease, lg is recommended to provide immediate protection against HA infection until an active response to the vaccine is produced. Given the lack of data to support benefit of Ig after 14 days, there is no recommendation for its use after this time period.

\section{Funding}

The work of $\mathrm{NACl}$ is supported by the Public Health Agency of Canada.

\section{Conflict of interest}

None.

\section{References}

1. National Advisory Committee on Immunization (NACl) Update on the Recommended us of Hepatitis A Vaccine. Ottawa ON: PHAC; 2016. http://www.healthycanadians. gc.ca/publications/healthy-living-vie-saine/hepatitis-a-vaccineupdate-recommended-use-2016-mise-a-jour-recommandationshepatite-a-vaccin/index-eng.php.

2. National Advisory Committee on Immunization (NACl). Canadian Immunization Guide: Part 4: Hepatitis A vaccine. Ottawa ON: PHAC; 2016 [updated 2016 Aug]. http:// www.healthycanadians.gc.ca/publications/healthy-livingvie-saine/4-canadian-immunization-guide-canadienimmunisation/index-eng.php?page $=6$. 